\title{
Space Charge Behavior of Quantum Dot-Doped Polystyrene Polymers

\author{
Zhipeng Lei
}

Taiyuan University of Technology

Shanxi Key Laboratory of Mining Electrical Equipment and Intelligent Control, College of Electrical and Power Engineering Taiyuan, Shanxi 030024 China

\author{
Davide Fabiani, Tommaso Bray and Chuanyang Li \\ University of Bologna \\ Department of Electrical, Electronic and Information Engineering 'G. Marconi' \\ Bologna, BO 40136, Italy
}

Xinyu Wang and Thomas Andritsch

University of Southampton

Tony Davies High Voltage Laboratory, Electronics and Computer Science

Southampton, SO17 1BJ, UK

\author{
Alberto Credi and Marcello La Rosa \\ University of Bologna and Consiglio Nazionale delle Ricerche \\ Center for Light Activated Nanostructures, Institute for Organic Synthesis and Photoreactivity \\ Bologna, BO 40129, Italy
}

\begin{abstract}
This paper deals with the role played by the interface and bulk volume of the nanofiller about affecting the electrical properties of a nanocomposite material. For this purpose, a simple and completely amorphous matrix, polystyrene (PS), is used as base material, and core-shell quantum dots are exploited for simulating the structure of nanocomposites: CdSe core and CdSe-ZnS core-shell semiconductor quantum dots (QDs) are added into a PS matrix. The latter is to highlight the effect of the $\mathrm{ZnS}$ interface and as contrast to the core material. Dispersion and distribution of QDs are first microscopically observed and optimized, by including isopropyl alcohol in the manufacturing phase as an additional solvent. Among electrical properties the focus is on space charge accumulation, tested by means of the pulsed electroacoustic technique at $10 \mathrm{kV} / \mathrm{mm}$ and $50 \mathrm{kV} / \mathrm{mm}$ on CdSe and CdSe-ZnS doped PS composites. Results are then compared with a reference PS without QDs. Trap depth and density are also obtained by space charge measurement results. When CdSe QDs are added to PS, the trap density increases with respect to the baseline values measured on the unfilled polymer. In contrast, the $\mathrm{ZnS}$ shell around the CdSe core creates an additional trap level with lower trap depth, which increases charge mobility, thus turning homocharge into heterocharge accumulation. Therefore, the surface shell-structure of QD nanocrystals appears to significantly influence the space charge behavior of the nanocomposite, independently of the polymer.
\end{abstract}

Index Terms - quantum dot (QD), polystyrene, polymer, space charge, nanocomposites

\section{INTRODUCTION}

NANOCOMPOSITES have been showing unexpected changes to bulk properties, i.e. permittivity, charge transport

\footnotetext{
Manuscript received on 27 October 2020, in final form xx Month 20yy,
} accepted xx Month 20yy. Corresponding author: D. Fabiani. behaviour, and the enhancement of key dielectric parameters like the breakdown strength and long-term ageing behaviour (treeing resistance). A number of models attempted to explain the effects observed in nanocomposites, e.g. the electrical double layer model [1], the multi-core model [2], an organic and inorganic composites hybrid network model [3], the polymer chain alignment model [4], the interphase volume model [5], the multi-region structure around spherical nanoparticles [6], the 
quantum dot model [7], and so on. Most of these models are based on the assumption that the molecular structure in the interphase layer of the polymer adjacent to the nanoparticle surfaces is subject to a reorganization, giving rise to an interphase volume. Therefore, surface treatment is seen as an effective technique to improve said interface region, affecting the properties of said interphase volume. Nanoparticles are typically treated with silane coupling agents to modify surface chemistry and thus interface tension [8]. However, recent work suggested that there could be also a particle interphase, not only a polymer interphase [9].

Quantum dots (QDs) are semiconductor nanocrystals with diameter in the range of $2-10 \mathrm{~nm}$ and can be coated by a thin shell. Because of their unique opto-electronic properties, affected by quantum confinement phenomena, they have been the subject of extensive investigations in different areas of research over the past two decades [10]. The interest in these materials has been primarily motivated by the possibility of tuning their electronic, optical, structural, and redox properties by changing the particle size and shape, without changing the chemical composition [11]. In addition, the surface of QDs can be modified by exploiting both self-assembly and ligand exchange methodologies. Therefore, based on the evidence of the charge size confinement and the Coulomb blockade affecting the permittivity and charge transport of polymer [7], fine-tuned QDs may be able to tailor polymer to achieve the desired function. Moreover, referring to T. Tanaka's QD models, if a nanoparticle is considered as a core and interface between nanoparticles and polymer is the shell, Core QDs and core-shell QDs can provide a method to highlight the effect of interphase region on electrical properties of nanocomposites, because the size and shape of QDs can also be precisely controlled by current nanofabrication technology [12].

In this work, QDs were mixed with a simple polymer system, in order to investigate the influence of interface/interphase region on the properties of the bulk material. Two types of QDs, CdSe core QDs and CdSe-ZnS core-shell QDs, both with a hydrophobic surface consisting of a monolayer of molecular ligands, were added in polystyrene (PS) by two different preparation methods. PS was selected since it is a simple and predictable polymer system, where extensive literature exists on its dielectric properties. The dispersion of nano composites was analyzed, as well as the space charge distribution at $10 \mathrm{kV} / \mathrm{mm}$ and $50 \mathrm{kV} / \mathrm{mm}$. Based on these results the trap distribution was calculated, in order to establish the effect of QD core and shell respectively, on the dielectric.

\section{METHODOLOGY}

\subsection{MATERIALS}

Spherical CdSe core quantum dots and CdSe-ZnS core-shell quantum dots were synthesized and characterized according to published procedures $[13,14]$. The surface of the resulting QDs is coated with a hydrophobic monolayer consisting of organic ligands - specifically, tri(n-octylphosphine)oxide, $\operatorname{tri}(n$ octyl)phosphine and $n$-hexadecylamine - that prevents aggregation in solution. It is well known that such a molecular layer constitutes an electrically and chemically insulating barrier, thereby contributing to the charge confinement by passivation of the semiconducting surface [15]. The diameter of $\mathrm{CdSe}$ core and the thickness of the $\mathrm{ZnS}$ shell, determined by optical spectroscopy [16], are reported in Table 1.

Table 1. Dimension of the quantum dots.

\begin{tabular}{c|c|c}
\hline Sample & $\begin{array}{c}\text { Core diameter } \\
(\mathrm{nm})\end{array}$ & $\begin{array}{c}\text { Shell thickness } \\
(\mathrm{nm})\end{array}$ \\
\hline CdSe core & 4.77 & - \\
CdSe-ZnS core-shell & 4.25 & 0.5 \\
\hline
\end{tabular}

QDs were blended with polystyrene, 430102 Aldrich, by a solvent blending method. In the preparation, two different preparation procedures were used in order to define which one would yield better results in terms of dispersion and distribution of the nanoparticles, as well as in the overall electrical properties of the composite material.

\subsection{SAMPLE PREPARATION - FIRST METHOD}

A quantity of $5 \mathrm{~g}$ of PS was dissolved in $25 \mathrm{ml}$ of dichloromethane (DCM) in an enclosed container at room temperature. Similarly, $5 \mathrm{mg}$ of QDs powder was dispersed in $3 \mathrm{ml}$ of DCM. After the PS pellets and QDs powder were well dissolved, the QDs/DCM mixture was added in PS/DCM mixture and mechanically stirred until it became gel-like. To further remove the solvent by evaporation, the mixture was placed in the fume cupboard for 4 days. The obtained composites were hot-pressed to remove larger bubbles, then dried in a vacuum oven at $60{ }^{\circ} \mathrm{C}$ for 3 days to remove the solvent residues. Finally, the composite was pressed into disc-like samples with a thickness of about $0.24 \mathrm{~mm}$ and with a filler content of $0.1 \mathrm{wt} \%$ of QDs.

\subsection{SAMPLE PREPARATION - SECOND METHOD}

For the second method, isopropyl alcohol (IPA) was used as a non-solvent to extract PS/QDs. QDs and PS were dissolved in DCM with initially the same procedure as described above, except for $4.995 \mathrm{~g}$ of PS was dissolved in $50 \mathrm{ml}$ of DCM in this method. The PS/QDs/DCM mixture was then poured into a beaker with $75 \mathrm{ml}$ IPA. The resulting precipitates were separated from the solvent mixture with a filter funnel, and placed in the fume cupboard to dry, before final application of hot press and vacuum similar to the first method. Finally, the resulting materials were pressed into the correct shape.

In this paper, undoped PS and PS doped with $0.1 \mathrm{wt} \%$ of core QDs, and core-shell QDs with or without the use of IPA were denoted as "PS", "PS-C-IPA", "PS-CS-IPA", "PS-C" and "PSCS", respectively. Table 2 is the sample nomenclature.

Table 2. Studied materials.

\begin{tabular}{cccc}
\hline Sample name & Filler & Processing & $\begin{array}{c}\text { Filler loading } \\
(\mathrm{wt} \%)\end{array}$ \\
\hline PS & - & - & 0 \\
PS-C & CdSe core & Without IPA & 0.1 \\
PS-CS & CdSe-ZnS core-shell & Without IPA & 0.1 \\
PS-C-IPA & CdSe core & With IPA & 0.1 \\
PS-CS-IPA & CdSe-ZnS core-shell & With IPA & 0.1 \\
\hline
\end{tabular}

\subsection{MORPHOLOGY MEASUREMENT}

Transmission electron microscopy (TEM) of PS/QDs composites was conducted by employing a Hitachi HT 7700 TEM. Sample slices for TEM with a thickness of $90 \mathrm{~nm}$ were 
obtained with the help of a Reichert Ultracut E ultramicrotomes.

\subsection{MEASUREMENT SETUP}

Space charge behavior of the samples was measured by the pulsed electroacoustic (PEA) technique at room temperature under applied electric fields between 10 and $50 \mathrm{kV} / \mathrm{mm}$. The anode was a semicon for improved acoustic impedance, and the aluminum cathode was treated with silicone oil. A voltage pulse with an amplitude of $500 \mathrm{~V}$ and a width of $10 \mathrm{~ns}$ was applied to the sample through a coupling capacitor. The sample was firstly polarized for 3 hours at $10 \mathrm{kV} / \mathrm{mm}$, and then depolarized until negligible charge remained. At $50 \mathrm{kV} / \mathrm{mm}$, the poling time was 12 hours and the depolarization time was more than 3 hours. Before the measurement at each electric field, the sample was shorted and discharged at $50{ }^{\circ} \mathrm{C}$ for more than 2 days in order to minimize the effect of potential residual charge.

\section{EXPERIMENTAL RESULTS}

\subsection{MORPHOLOGY}

Representative TEM results of PS-C and PS-C-IPA are shown in Figure 1. It can be observed in Figure 1a that QDs in the PSC sample synthesized via the first method lead to quite distinct agglomerations, with dimension in the micrometer range. The agglomerations are gathered by clusters of QDs with the diameter about $20 \mathrm{~nm}$ as shown in Figure 1b. In contrast, PS-CIPA shows a very good distribution of the QDs, as can be observed in Figure 1c. The average diameter of QD clusters is about $20 \mathrm{~nm}$, which suggests that about several QDs (the morphology of two types of QDs are shown in Figure 1d) consists of the QD cluster dispersing in PS. While the second method leads to a small loss of material during preparation, the method using IPA as a non-solvent leads to significantly better results in terms of nanoparticles' dispersion and distribution.

\subsection{SPACE CHARGE BEHAVIOUR AT $10 \mathrm{KV} / \mathrm{MM}$}

All the manufactured samples were tested at $10 \mathrm{kV} / \mathrm{mm}$ in order to compare the effect of dispersion of core and core-shell DQs on space charge accumulation features. Space charge distribution was measured for the reference PS, PS-C-IPA, and PS-CS-IPA. Space charge profiles of PS are shown in Figure 2. During poling (voltage-on), a small amount of homocharge injection can be seen near both the anode and the cathode. This injection charges can be further verified by the depolarization phase as presented in Figure 2b. Homocharge accumulates in the vicinity of electrodes and dissipates slowly. After 1 hour there are still measurable amounts of charges with the maximum charge density of $1.8 \mathrm{C} / \mathrm{m}^{3}$ staying in the bulk.

The space charge properties of PS composites with $0.1 \mathrm{wt} \%$ of CdSe core QDs prepared with the first method (no IPA) are shown in Figure 3. Similar to the reference PS sample, homocharge is injected from both electrodes. Some heterocharge, about $0.6 \mathrm{C} / \mathrm{m}^{3}$, can be found immediately in the vicinity of the anode, which may be due to the QDs agglomerations which act as impurities and effectively charge trapping sites. During depoling (voltage-off), homocharge with the density of about $1.2 \mathrm{C} / \mathrm{m}^{3}$ near the cathode is evident and is
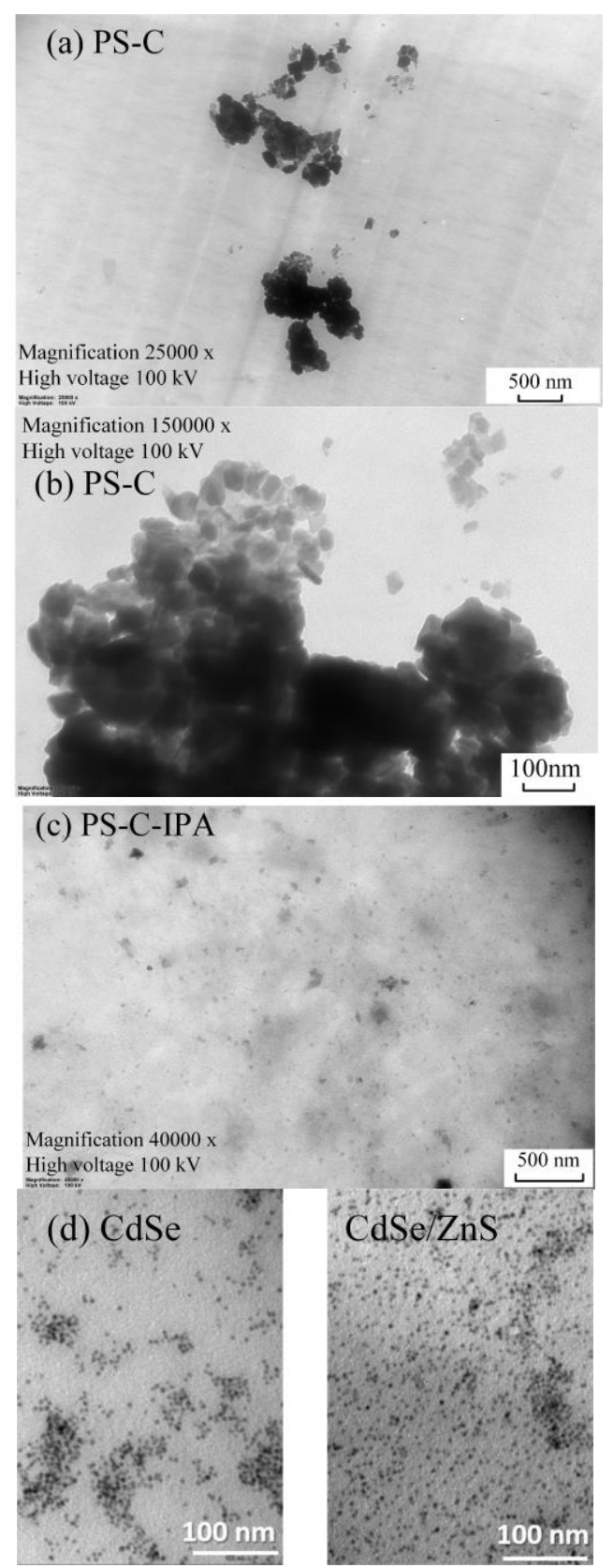

Figure 1. TEM images of: (a) and (b) PS-C, (c) PS-C-IPA, (d) quantum dots.

retained in the bulk, just as seen for the reference PS.

The accumulated heterocharge dissipates very fast within the initial $20 \mathrm{~s}$. This heterocharge build-up is not likely to be originated from the migration of homocharge injected from the cathode. First of all, heterocharge canbe immediately observed when an electric field is applied and decays fast as soon as the voltage is removed. In this case, samples may have impurities resulting in the charge accumulating near the anode [17]. Secondly, if heterocharge comes from the injection of the negative electrode, its quantity will increase with poling time. As shown in Figure 3c, the quantity of heterocharge in the bulk from $90 \mu \mathrm{m}$ to $190 \mu \mathrm{m}$ rises to about $-3.5 \mathrm{nC}$ after poling about 1 hour, but it remains about $-3.7 \mathrm{nC}$ even though the poling time was extended to 12 hours. Oppositely, the quantity of injected charge in the vicinity of cathode slowly increases with poling time. 

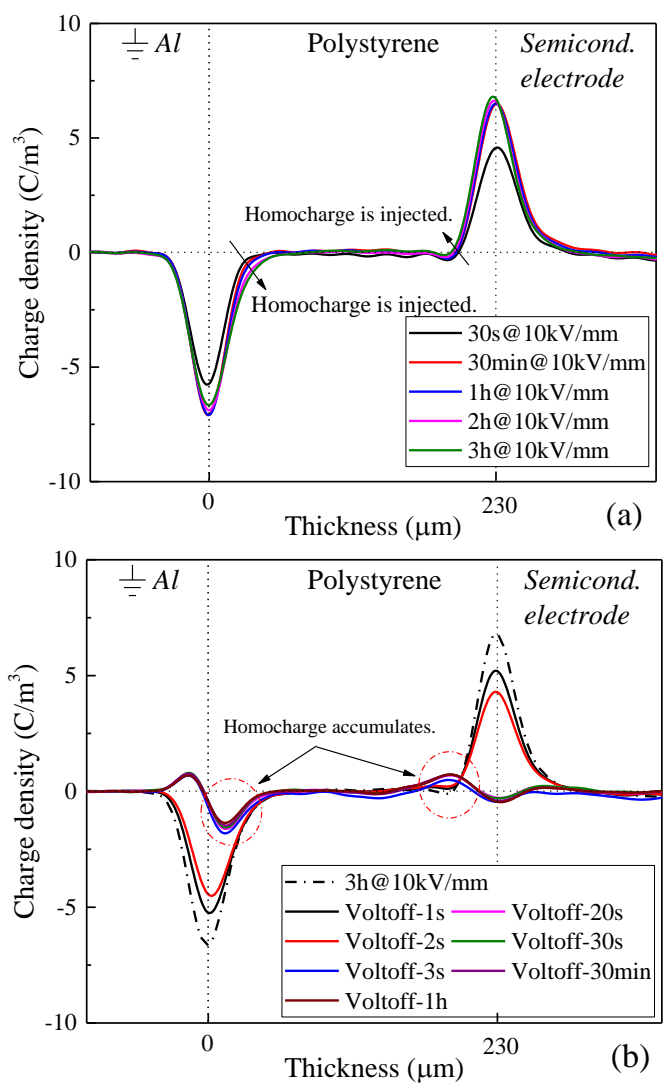

Figure 2. Space charge profiles of PS at $10 \mathrm{kV} / \mathrm{mm}$ : (a) polarization, (b) depolarization.

In Figure 4 the space charge profiles of PS filled with the 0. $1 \mathrm{wt} \%$ of CdSe core quantum dots are plotted, using the second synthesis method (with IPA). Based on these results the conclusion can be drawn that either the better dispersion of QDs improves the space charge distribution, or the IPA altered the density of available trapping sites at the particle/polymer interface. During poling, homocharge, about $-2.5 \mathrm{nC}$, is injected from the cathode. There is much suppressed positive charge accumulation in the vicinity of the anode. The space charge distribution during depoling provides evidence clearer picture in that no significant amount of charges accumulate in the bulk. It is thus quite clear that the preparation method has a profound effect on the charge dynamics of these QD-composites, with quite significant differences to the dielectric properties already at low electric fields.

\subsection{SPACE CHARGE BEHAVIOUR AT $50 \mathrm{KV} / \mathrm{MM}$}

In this section, space charge results obtained on samples showing the best dispersion, e.g. PS-C-IPA and PS-CS-IPA at high fields of $50 \mathrm{kV} / \mathrm{mm}$, are discussed and compared with the reference PS for understanding the influence of core and shell on the space charge properties.

The space charge profiles of the reference PS at $50 \mathrm{kV} / \mathrm{mm}$ are shown in Figure 5. When the electric field is increased to this level, negative charges can be easily injected from the cathode, while there is an insignificant positive charge injection at the anode. Similar phenomena have been widely reported with different types of polymers, such as polyethylene [18], epoxy resin [19], and polyethylene-graft-polystyrene/LDPE composites [20]. In general, the higher mobility of electrons compared to holes under electric field has been cited as main reason [18]. When Lewis investigated the charge transport in semi-crystalline polyethene, he concluded that electrons had higher mobility than holes, as electrons can move more freely at inter-chain spaces within the amorphous region of polymers [21]. Thus, when space charge properties of non-crystalline polymer like PS with an amorphous structure are studied, negative charges can be more easily injected in the bulk, because the abovementioned high electron mobility under high electric fields, while holes are more restricted. With the increase of poling time, the injected depth of negative charges increases. After about 12 hours, injected negative charges nearly
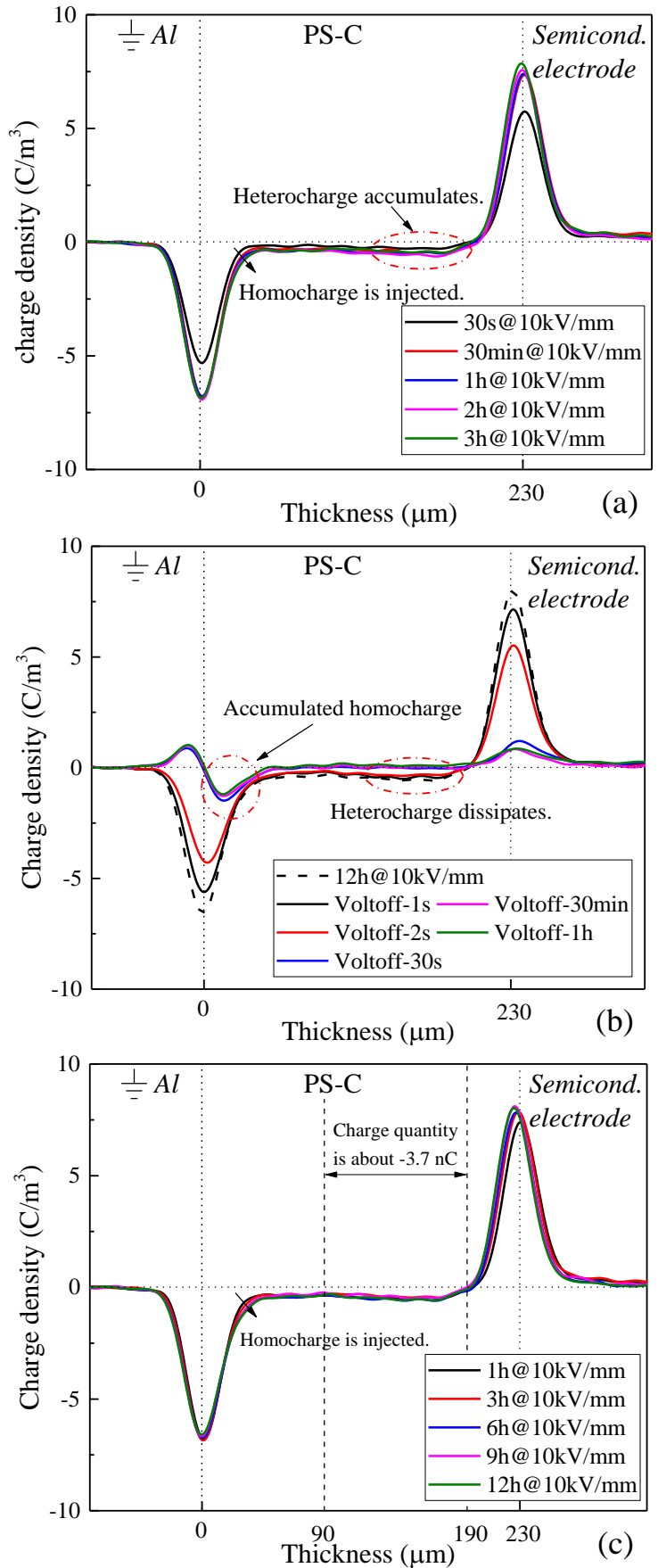

Figure 3. Space charge profiles of PS-C at $10 \mathrm{kV} / \mathrm{mm}$ : (a) polarization, (b) depolarization, (c) polarization for 12 hours. 

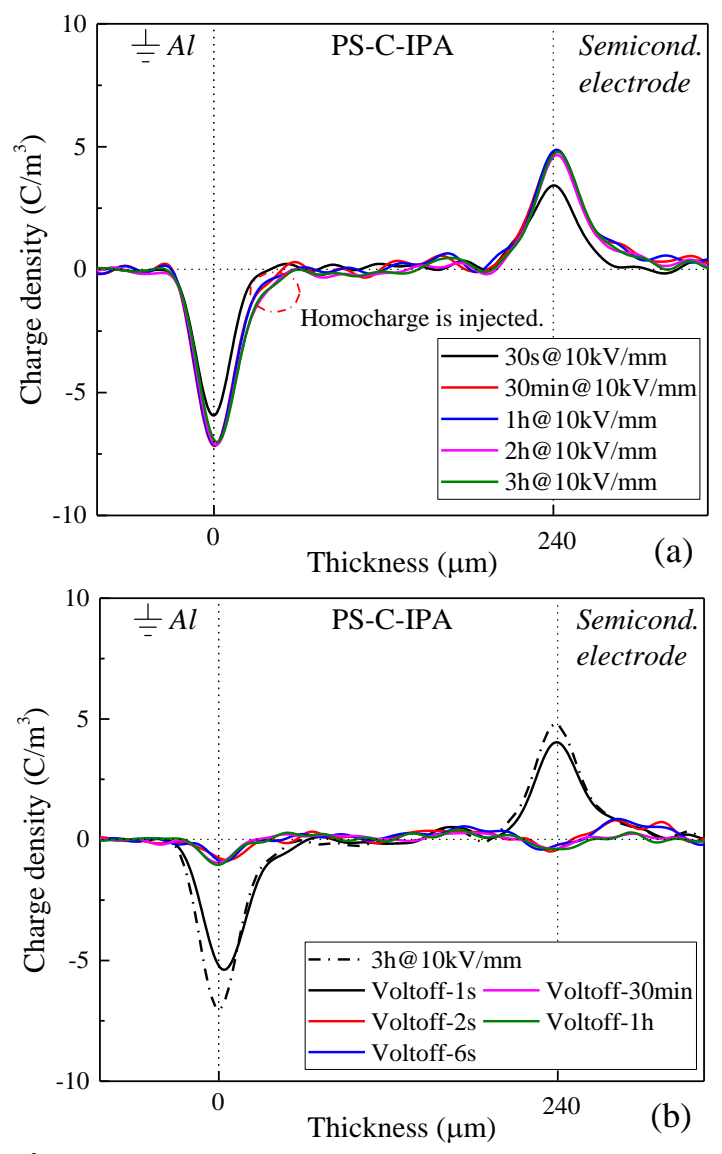

Figure 4. Space charge profiles of PS-C-IPA at $10 \mathrm{kV} / \mathrm{mm}$ : (a) polarization, (b) depolarization.

reaches the anode. During depoling, as shown in Figure 5b, the injected homocharge remains in the bulk even after about 6 hours, and the charge density is still high, about $-8 \mathrm{C} / \mathrm{m}^{3}$. When the injection depth of negative charges in bulk increases, the electric field close near cathode will lower, but it will increase to approximately $70 \mathrm{kV} / \mathrm{mm}$ at the contact point of positive and negative charge in the front of the cathode. In this case, insulation will bear higher stress.

Figure 6 displays the space charge profiles of PS-C-IPA at $50 \mathrm{kV} / \mathrm{mm}$. Comparing with PS, homocharge is injected into PS-C-IPA from both electrodes. The injection depth of positive charges is deeper than negative charges. The charges accumulating in the bulk will dramatically increase the electric field. Both positive and negative charges dissipate very slowly during depolarization. After depoling 6 hours as shown in Figure $6 \mathrm{~b}$, the quantity of negative charge is about $-45 \mathrm{nC}$, and positive charge is about $37 \mathrm{nC}$. Therefore, the space charge distribution is closely related with the applied electric field besides the blending of QDs. When the dispersion of CdSe core QDs is good, more positive charges can also be injected and migrate in bulk at high electric field.

The space charge profiles of PS doped with CdSe-ZnS coreshell QDs at $50 \mathrm{kV} / \mathrm{mm}$ are reported in Figure 7. During poling phase, heterocharge accumulates gradually in the vicinity of cathode, which can also be found near anode in contrast to that in PS-C-IPA. It rises to about $20 \mathrm{nC}$ after poling for 12 hours. Heterocharge near cathode can slightly increase the electric field in this area. Negative charges, which is about $-23 \mathrm{nC}$
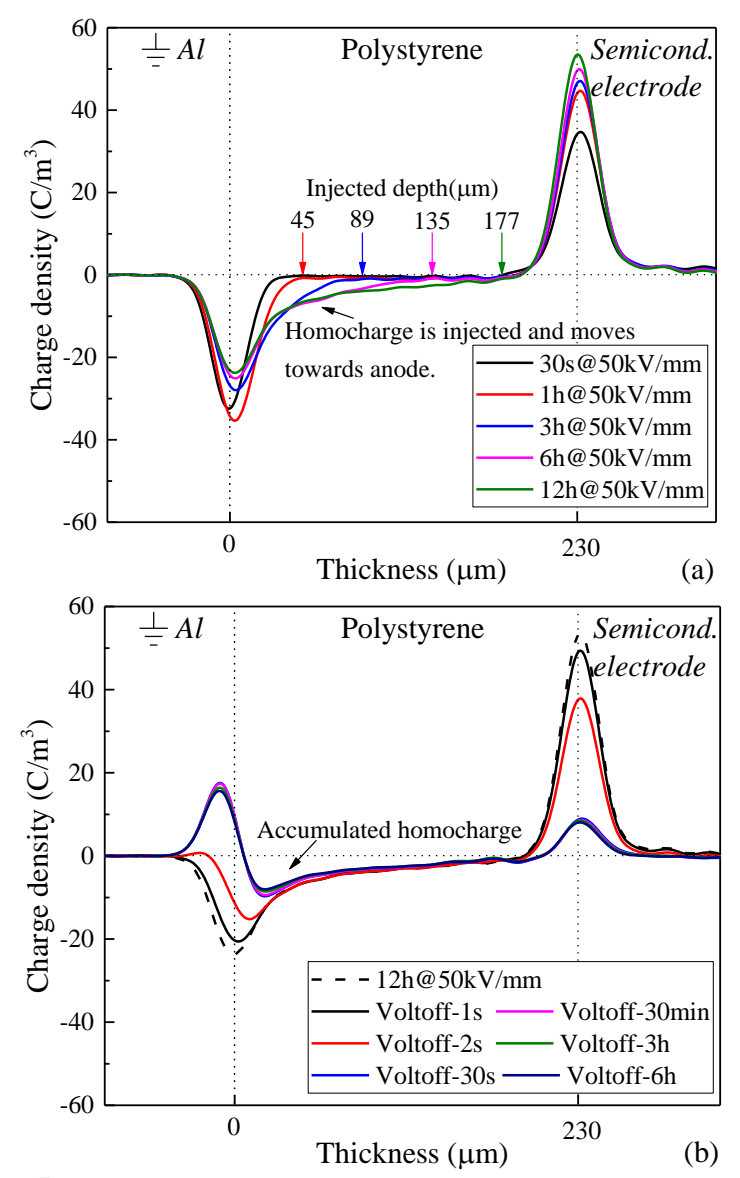

Figure 5. Space charge profiles of PS at $50 \mathrm{kV} / \mathrm{mm}$ : (a) polarization, (b) depolarization.

(calculated on the basis of the depoling profile in an hour), are extracted from cathode, which can be evidently seen during depolarization phase. Both heterocharge and homocharge in the bulk are difficult to be totally dissipated.

\section{DISCUSSION}

The space charge distribution in the insulation at DC electric field depends on the trap distribution that can be obtained based on the isothermal decay current method. The dependency of the current density $j(t)$ on the surface potential $V_{s}(t)$ can be expressed as:

$$
\begin{aligned}
& j_{\text {decay }}(t)=\frac{C}{A} \frac{\mathrm{d} V_{s}(t)}{\mathrm{d} t} \\
& C=\varepsilon_{0} \varepsilon_{r} A / d
\end{aligned}
$$

where $C$ is the equivalent capacitance of the sample, $A$ and $d$ are the surface area and thickness of sample, respectively.

Moreover, the surface space charge density $\sigma(t)$ is

$\sigma(t)=\varepsilon_{0} \varepsilon_{r} V_{s}(t) / r^{\prime}$

where $r^{\prime}$ is the mean injection depth of charges.

If $r^{\prime}$ is assumed to be a selected region $\Delta x$, no more than about $100 \mu \mathrm{m}$, from electrode interface to insulation as shown in Figure 8, the surface space charge density can be expressed as: 

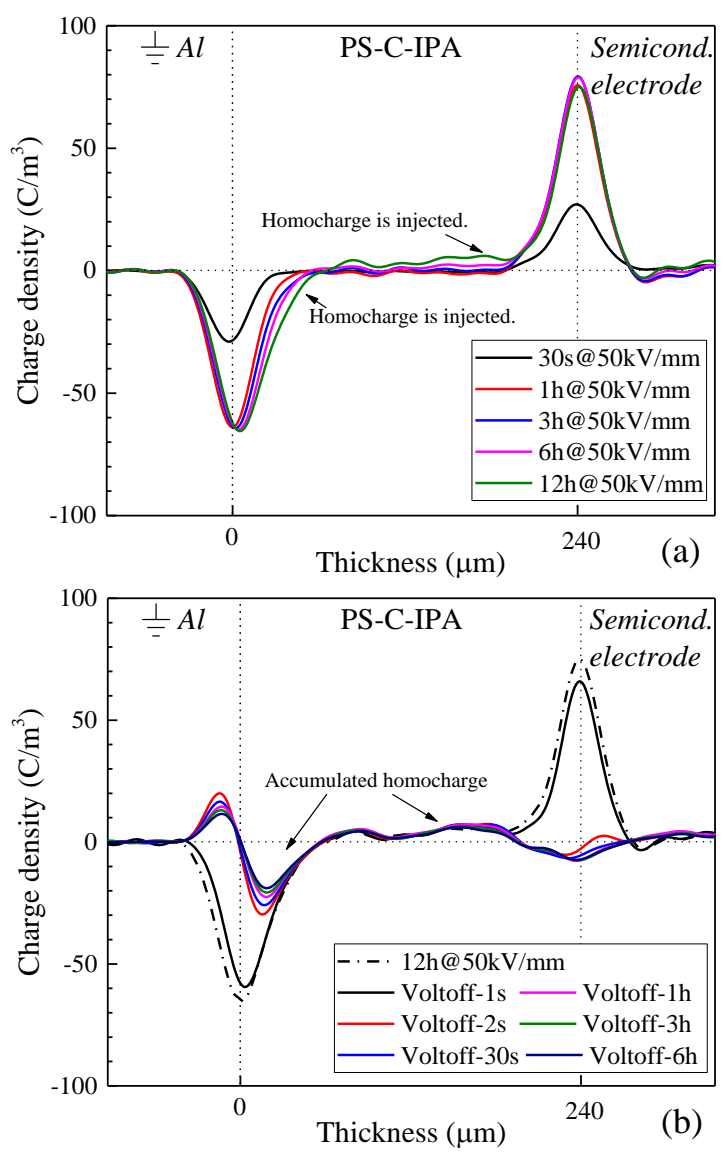

Figure 6. Space charge profiles of PS-C-IPA at $50 \mathrm{kV} / \mathrm{mm}$ : (a) polarization, (b) depolarization.

$$
\sigma(t)=\int_{0}^{\Delta x}\left|\rho_{\mathrm{dp}}(t, x)\right| \mathrm{d} x
$$

where $\rho_{\mathrm{dp}}(t)$ is the space charge volume density of insulation during depolarization phase; $\Delta x$ can be equal to $\Delta x_{p}$ or $\Delta x_{n}$, depending on which charge (positive or negative) is considered.

The decay characteristic of positive or negative space charge density can be fitted by a double exponential function, so the relationship between decay current density and charge density of space charge during depolarization can be obtained by (1) to (4) as:

$$
j_{\text {decay }}(t)=\frac{\Delta x}{d} \frac{\mathrm{d} \sigma(t)}{\mathrm{d} t}
$$

where $r^{\prime}$ is equal to $\Delta x$.

Then, the trap depth, $E_{t}$, and trap energy density, $N\left(E_{t}\right)$, can be calculated by:

$$
\begin{aligned}
& E_{t}=k T \ln v t \\
& N\left(E_{t}\right)=\frac{2 t}{q d k T} f_{0}^{-1}\left(E_{t}\right) j_{\text {decay }}(t)
\end{aligned}
$$

where $k$ is Boltzmann's constant, which is $8.617 \times 10^{-5} \mathrm{eV} / \mathrm{K}, T$ is absolute temperature, $v$ is de-trapping attempt frequency which is $k T / h, h$ is Planck constant. It should be noted that the trap distribution calculated according to the above method is the trap depth of trapped charges and does not reflect the entire trap distribution in the material.
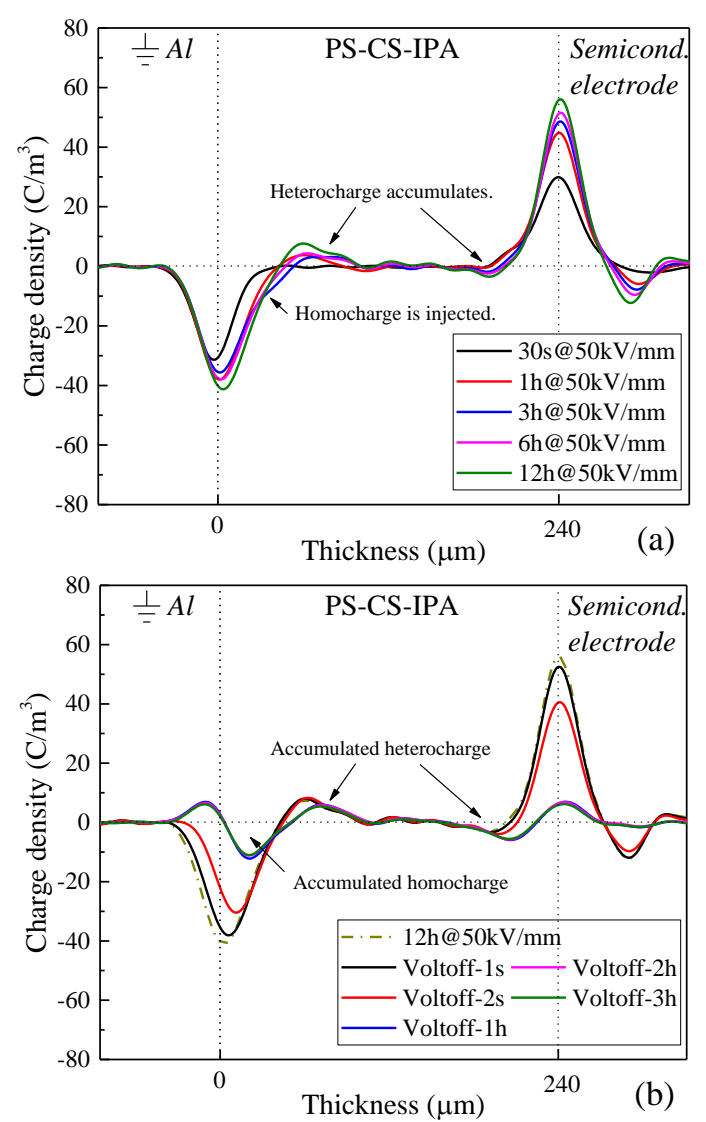

Figure 7. Space charge profiles of PS-CS-IPA at $50 \mathrm{kV} / \mathrm{mm}$ : (a) polarization, (b) depolarization.

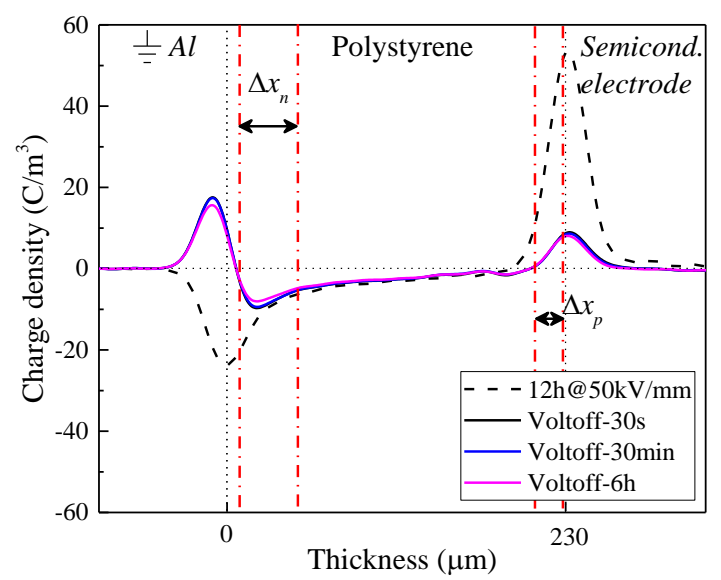

Figure 8. Selected region of the mean injection depth of charges.

Figure 9 shows the trap distribution obtained from the depolarization of space charge tested after poling 12 hours at $50 \mathrm{kV} / \mathrm{mm}$. The trap level of PS is about $1 \mathrm{eV}$, which is very close to that of PS-C-IPA, about $0.98 \mathrm{eV}$. However, the trap density of PS-C-IPA is highest, which means that more charges can be trapped. By contrast, the presence of the shell in the QDs (PS-CS-IPA), decreased trap depth to about $0.94 \mathrm{eV}$, slightly lower than reference PS and PS doped with core quantum dots.

It can be further analyzed from Figure 10, which is calculated from the decay characteristic of positive and negative space charge accumulating near the electrode separately. This method can remove the effect of bulk charges on the trap distribution. It can be seen in PS whether is doped with quantum dots that 
the traps with relative high trap level exists, which can trap both positive and negative charges. However, when CdSe core quantum dots are dispersed in PS, the density of traps blocking negative charges increases, as the trap distribution of PS-C-IPA shows in Figure 10b. It should be noted that the trap distribution of positive charge in both PS-C-IPA and PS-CS-IPA was calculated from the positive charge in the bulk, because there is no evidence of positive charges trapped at the interface of anode. The positive charges at the anode interface dissipate near instantaneously, thus they were not considered.

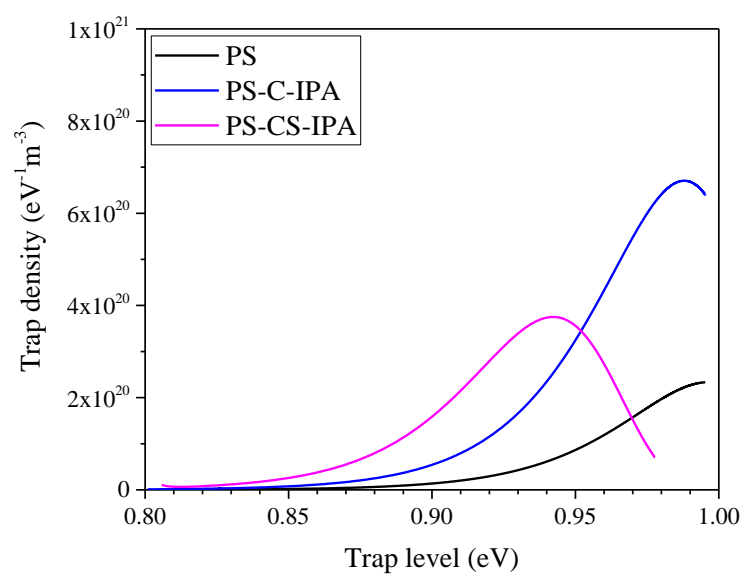

Figure 9. Trap distribution of different samples.

The PS-CS-IPA sample with CdSe-ZnS core-shell QDs exhibits two levels of deep traps which is different with other samples. The lowest trap level of PS-CS-IPA near both electrodes is about $0.83 \mathrm{eV}$. In this case, charges will be able to de-trap and move into the bulk, which may explain why there are lots of heterocharge accumulating near both electrodes.

The energy band gaps of PS, $\mathrm{ZnS}$ and $\mathrm{CdSe}$ are about $5 \mathrm{eV}$, $3.6 \mathrm{eV}$ and $2.1 \mathrm{eV}$ respectively [22]. Therefore, referring to reports in the ref. [7, 10, 23], the electron transfer process of PS doped with QDs at the high electric field of $50 \mathrm{kV} / \mathrm{mm}$ is illustrated based on the simplified energy level diagram as shown in Figure 11. In the PS-C-IPA, there are two kinds of transfer processes: (1) under high electric field, electrons jumping over or tunnelling potential barriers from the cathode possibly detrap from the deep traps (about $0.9 \mathrm{eV}$ which is the difference of the lowest unoccupied molecular orbital (LUMO) of PS and the conduction band of CdSe) and drift slowly through PS [24], which results in the injection of negative charge in the bulk; (2) electrons in PS are trapped and confined in QDs because of the conduction band energy of PS is higher than CdSe QDs, which suppresses the migration of negative charges towards the anode and is a reason of the increasing of trap density.

Calculated by results of the depolarization characteristic on the method reported in [25], the charge mobility of PS-CS-IPA and PS-C-IPA is about $10^{-15} \mathrm{~m}^{2} /(\mathrm{V} \cdot \mathrm{s})$ and $10^{-16} \mathrm{~m}^{2} /(\mathrm{V} \cdot \mathrm{s})$. Therefore, shallower traps may be introduced by the shell, which contributes to the drift of injected charges. Combining with the poling time, no less than 12 hours, a part of injected charges in PS-CS-IPA bulk has the enough time to transfer to the opposite electrode under the high electric field. When coreshell QDs are doped in PS: (1) the electrons in PS can hop into the surface traps of $\mathrm{ZnS}$ shell and then de-trap because the
LUMO of PS is close to the conduction band of $\mathrm{ZnS}$. Therefore, the shell can provide routes for the charge transfer and results in the relative shallow traps in PS-CS-IPA. (2) Electrons from the valence band can rapidly relax to the empty traps at the $\mathrm{ZnS}$ shell surface [10] and hop into the deep traps in PS. Both 1 and 2 contribute to the migration of electrons, which provides one explanation on the appearance of heterocharge gradually accumulating in the vicinity of cathode in PS-CS-IPA.
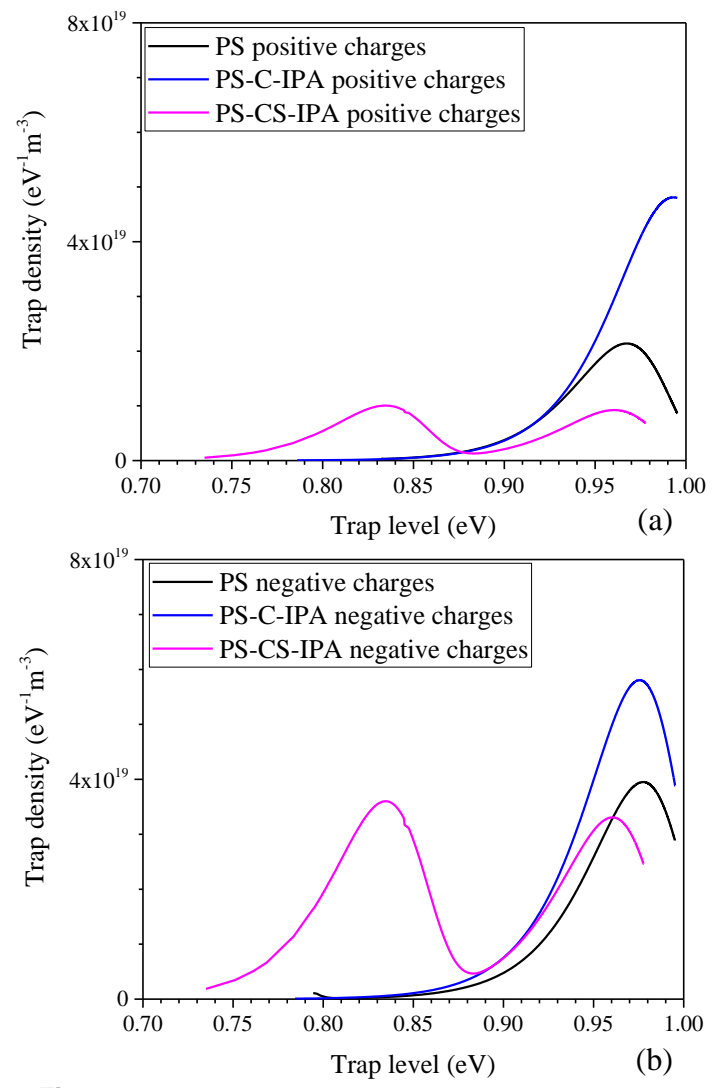

Figure 10. Trap distribution near: (a) anode, (b) cathode.
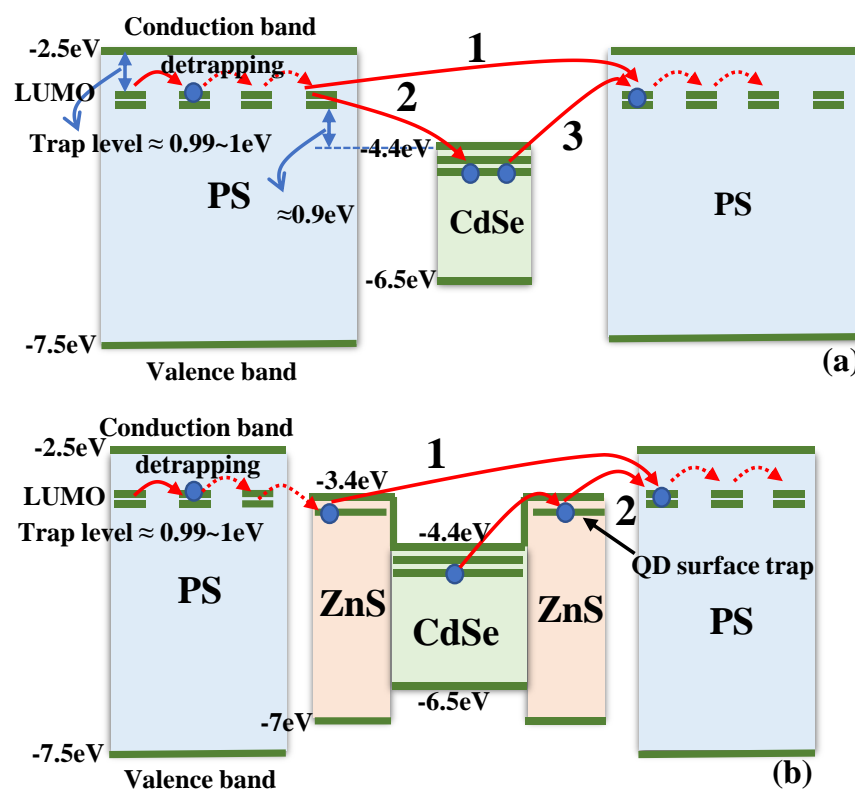

Figure 11. Simplified energy level diagram of PS (a) with core QDs, (b) with core-shell QDs. 


\section{CONCLUSIONS}

In this paper, core-shell QDs are used to analyze which feature of nanoparticles, i.e. bulk and/or interface area or interphase volume, dominates the behavior of a nanocomposite, with focus on the space charge properties. At high electric fields, around $50 \mathrm{kV} / \mathrm{mm}$, a significant amount of space charge is injected into all samples from the electrodes. In the reference PS, negative charges injected from cathode move very slowly towards the opposite electrode. In the case of nanocomposites, the introduction of core QD particles increases the trap density, thus we could observe the amount of charges, particularly homocharge, injected from both electrodes to increase. In contrast to the core-QD behavior, core-shell QDs nanocomposites exhibit heterocharge accumulating near both electrodes. This can be attributed to the lower energy traps introduced by the $\mathrm{ZnS}$ shell, where injected charges can transfer to the opposite electrode under the high electric field, thus forming heterocharge, when the poling time is long enough.

Therefore, controlling both core and shell of the particles, space charge properties of the polymer can be affected, and effectively tailored. Although they both influence the space charge behavior, the shell of QDs represents the dominant factor. These findings support the hypothesis that the interface between nano particles and matrix is the key issue in determining the space charge behavior of an electrical insulating material. In particular, the outer layer, here represented by the $\mathrm{ZnS}$ shell, can be of a quite different structure to the nominal core-material of commercially available nanoparticles. Such a strong effect of the particle surface could also explain the differences often found in literature as regards space charge behavior of materials having similar nanoparticles. Depending on processing and preparation, indeed, the surface of such particles can be different and this will not only impact filler dispersion/distribution, but also trap depth and density of the filler, thus leading to space charge results that can look very different, despite having nominally the same combination of particle and polymer. This can be considered both an advantage but also a major drawback of using nano-additives: on one hand, particle surface functionalization can help in tailoring space charge features, but on the other hand, contaminants, like by-products of the particle synthesis, moisture, surfactants or surface treatments can also lead to unexpected variations of the dielectric properties. This issue needs to be considered carefully, when designing a nanocomposite insulation system.

\section{ACKNOWLEDGMENT}

The authors would like to thank the generous help of associate Professor Maria Letizia Focarete in the Department of Chemistry "Giacomo Ciamician" of University of Bologna for providing the preparing instrument. This work was supported by the National Natural Science Foundation of China under Grant No. 51977137.

\section{REFERENCES}

[1] T. Lewis, "Interfaces: nanometric dielectrics," J. Phys. D: Appl. Phys., vol. 38, no. 2, pp. 202, Jan. 2005.

[2] T. Tanaka, "Dielectric nanocomposites with insulating properties," IEEE Trans. Dielectr. Electr. Insul., vol. 12, no. 5, pp. 914-928, Oct. 2005.

[3] J. Wen and G. L. Wilkes, "Organic/Inorganic Hybrid Network Materials by the Sol-Gel Approach," Chem. Mater., vol. 8, no. 8, pp. 1667-1681, Jan. 1996.

[4] T. Andritsch et al, "Proposal of the polymer chain alignment model," IEEE Int. Conf. Electr. Insul. Dielect. Phenom. (CEIDP), 2011, pp. 624-627.

[5] S. Raetzke and J. Kindersberger, "Role of interphase on the resistance to high-voltage arcing, on tracking and erosion of silicone/SiO2 nanocomposites," IEEE Trans. Dielectr. Electr. Insul., vol. 17, no. 2, pp. 607-614, Apr. 2010.

[6] S. Li et al, "Short-term breakdown and long-term failure in nanodielectrics: a review," IEEE Trans. Dielectr. Electr. Insul., vol. 17, no. 5, pp. 15231535, Oct. 2010.

[7] T. Tanaka, "A quantum dot model for nanoparticles in polymer nanocomposites," IEEE Trans. Dielectr. Electr. Insul., vol. 26, no. 1, pp. 276-283, Jan. 2019.

[8] Y. Xie et al, "Silane coupling agents used for natural fiber/polymer composites: A review," Compos. Part A - Appl. Sci., vol. 41, no. 7, pp. 806-819, Jul. 2010.

[9] F. N. Alhabill et al, "Introducing particle interphase model for describing the electrical behaviour of nanodielectrics," Mater. Des., vol. 158, no. 2018, pp. 62-73, Nov. 2018.

[10] M. Amelia et al, "Electrochemical properties of CdSe and CdTe quantum dots," Chem. Soc. Rev., vol. 41, no. 17, pp. 5728-5743, Jul. 2012.

[11] J. Weaver et al, "Synthesis and characterization of quantum dot-polymer composites,” J. Mater. Chem., vol. 19, no. 20, pp. 3198-3206, Mar. 2009.

[12] L. P. Kouwenhoven et al, "Few-electron quantum dots," Rep. Prog. Phys., vol. 64, no. 6, pp. 701-736, May 2001.

[13] M. La Rosa et al, "An Efficient Method for the Surface Functionalization of Luminescent Quantum Dots with Lipoic Acid Based Ligands," Eur. J. Inorg. Chem., vol. 2017, no. 44, pp. 5143-5151, Sept. 2017.

[14] M. La Rosa et al, “Designed Long - Lived Emission from CdSe Quantum Dots through Reversible Electronic Energy Transfer with a Surface Bound Chromophore," Angew. Chem. Int. Ed., vol. 130, no. 12, pp. 31583161, Jan. 2018.

[15] M. A. Boles et al, "The surface science of nanocrystals," Nat. Mater., vol. 15, no. 2, pp. 141-153, Feb. 2016.

[16] W. W. Yu et al, "Experimental Determination of the Extinction Coefficient of CdTe, CdSe, and CdS Nanocrystals," Chem. Mater., vol. 15, no. 14, pp. 2854-2860, Jul. 2003.

[17] M. Fu et al, "Influence of thermal treatment and residues on space charge accumulation in XLPE for DC power cable application," IEEE Trans. Dielectr. Electr. Insul., vol. 14, no. 1, pp. 53-64, Feb. 2007.

[18] Z. Li et al, "Trap Modulated Charge Carrier Transport in Polyethylene/Graphene Nanocomposites," Sci. Rep., vol. 7, no. 1, pp. 1-8, Jun. 2017.

[19] C. Li et al, "The potentially neglected culprit of DC surface flashover: electron migration under temperature gradients," Sci. Rep., vol. 7, no. 1, pp. 1-11, Jun. 2017.

[20] S. Song et al, "Enhanced Electrical Properties of Polyethylene-GraftPolystyrene/LDPE Composites," Polymers, vol. 12, no. 1, pp. 1-16, Jan. 2020.

[21] T. J. Lewis, "Polyethylene under electrical stress," IEEE Trans. Dielectr. Electr. Insul., vol. 9, no. 5, pp. 717-729, Dec. 2002.

[22] E. Khodabakhshi et al, "Suppression of electron trapping by quantum dot emitters using a grafted polystyrene shell," Mater. Horiz., vol. 6, no. 10, pp. 2024-2031, Jun. 2019.

[23] M. R. Hummon et al, "Measuring charge trap occupation and energy level in $\mathrm{CdSe} / \mathrm{ZnS}$ quantum dots using a scanning tunneling microscope," Phys. Rev. B, vol. 81, no. 11, pp. 115439, Mar. 2010.

[24] T. Takada, "Studies on Space Charge Accumulation Properties in Dielectric Materials," Proc. 2nd Int. Conf. Elect. Mater. Power Equip. (ICEMPE), 2019, pp. 1-8.

[25] G. C. Montanari et al, "Space-charge trapping and conduction in LDPE, HDPE and XLPE," J. Phys. D: Appl. Phys., vol. 34, no. 18, pp. 2902-2911, Sept. 2001. 